OPEN ACCESS

Edited by:

Yurong Lai,

Bristrol-Myers Squibb, USA

Reviewed by:

Viktória Jeney,

University of Debrecen, Hungary

Jing Lin,

Sunovion Pharmaceuticals, USA

${ }^{*}$ Correspondence:

Guoqiu Wu

nationball@163.com:

Naifeng Liu

Liunaifeng1962@126.com

Specialty section:

This article was submitted to Drug Metabolism and Transport,

a section of the journa

Frontiers in Pharmacology

Received: 11 January 2016 Accepted: 22 February 2016 Published: 08 March 2016

Citation:

Qu Q, Liu Y, Yan X, Fan X, Liu N and Wu G (2016) A Novel Pentapeptide Targeting Integrin $\beta 3$-Subunit Inhibits

Platelet Aggregation and Its Application in Rat for Thrombosis Prevention. Front. Pharmacol. 7:49. doi: 10.3389/fphar.2016.00049

\section{A Novel Pentapeptide Targeting Integrin $\beta 3$-Subunit Inhibits Platelet Aggregation and Its Application in Rat for Thrombosis Prevention}

\author{
Qingrong $\mathrm{Qu}{ }^{1}$, Yamin $\mathrm{Liu}^{2,3}$, Xuejiao Yan ${ }^{1}$, Xiaobo Fan ${ }^{2}$, Naifeng Liu ${ }^{1 *}$ and Guoqiu Wu ${ }^{2 *}$ \\ ${ }^{1}$ Department and Institute of Cardiology, Zhongda Hospital, Medical School of Southeast University, Nanjing, China, ${ }^{2}$ Center \\ of Clinical Laboratory Medicine of Zhongda Hospital, Institute of Biotechnology and Clinical Pharmacy, Southeast University, \\ Nanjing, China, ${ }^{3}$ Pharmacy Department of Zhongda Hospital, Southeast University, Nanjing, China
}

Background: Antiplatelet therapy plays a pivotal role in the prevention and treatment of thrombotic diseases. We reported the screening of P1C as a novel integrin-binding peptide from the C-terminal of connective tissue growth factor. Primary study indicated that $\mathrm{P} 1 \mathrm{C}$ has potential against platelet aggregation.

Objectives: We aimed to find the shortest active unit from the P1C fragments and explore its in vivo and in vitro activities.

Methods: A series of truncated P1C fragments was prepared and screened for antiplatelet activity. The most active fragment was evaluated using coagulation assays. Flow cytometry and confocal microscopy were used to determine the interaction between the peptide and the integrin. The in vivo potential was further explored using two types of rat models.

Results: From a series of truncated P1C forms, a so-called P1Cm peptide of 5-amino acids, namely, IRTPK was screened out as the shortest active unit with superior activity. Coagulation experiments and an in vivo toxicity assay demonstrated that $\mathrm{P} 1 \mathrm{Cm}$ is safe in vivo and inhibits ADP- and TH-induced human platelet aggregation in vitro in a concentration-dependent manner. Furthermore, it has limited effect on the coagulation parameters. Flow cytometry and confocal microscopy experiments consistently indicated that the peptide specifically binds the $\beta 3$-subunit of integrin on platelets. Further experiments using rat models of artery-vein shunt and carotid arterial thrombosis illustrated that P1Cm can effectively prevent thrombosis formation.

Conclusion: $\mathrm{P} 1 \mathrm{Cm}$ may be a new, promising antithrombotic alternative to currently available antiplatelet treatments.

Keywords: peptide, platelet, integrin beta3, blood coagulation, thrombosis

\section{INTRODUCTION}

Platelet activation, aggregation, and adhesion are considered the general pathogenesis of various cardiovascular disorders, such as acute coronary syndrome, myocardial infarction (De Luca, 2012), stroke, and unstable angina (Rodrigues et al., 2013). These pathological processes are mainly mediated by the receptor of glycoprotein IIb/IIIa or by integrin $\alpha \operatorname{IIb} \beta 3$ of the platelets 
(Liu et al., 2005). Each platelet contains 60,000-80,000 molecules of $\alpha \operatorname{IIb} \beta 3$ protein complexes (Armstrong and Peter, 2012).

Upon platelet activation, $\alpha \operatorname{IIb} \beta 3$ shifts into a high affinity conformation that efficiently binds the ligands and leads to platelet aggregation, which is considered the classic mechanism of action for all known platelet agonists (Lefkovits et al., $1995)$. Consequently, blocking the integrin $\alpha \operatorname{IIb} \beta 3$ receptor is an excellent strategy for the therapeutic intervention for thrombotic diseases (Di Mario, 2014).

Conventional $\alpha \operatorname{IIb} \beta 3$ inhibitors have helped establish the protocol for antiplatelet therapy in percutaneous coronary intervention (Hanna et al., 2010). Thus far, the United States Food and Drug Administration has approved three $\alpha \operatorname{IIb} \beta 3$ inhibitors: abciximab, eptifibatide, and tirofiban. However, retrospective analyses obtained through different means have indicated a high risk of bleeding arising from the administration of $\alpha \operatorname{IIb} \beta 3$ inhibitors (Hechler et al., 2011). Given the importance of antithrombotics in the treatment of ischemic disorders, additional efforts should be directed to searching for novel and better antithrombotic agents.

$\mathrm{P} 1 \mathrm{C}$ is a fragment from the C-terminal of connective tissue growth factor, which binds to various integrins such as $\alpha \nu \beta 3$ from endothelial cells (Cornel et al., 2015), $\alpha$ IIb $\beta 3$ from blood platelets, and alpha(6)-beta(1) from fibroblast cells (Gao and Brigstock, 2004). The nanoprobe prepared by conjugating P1C with ultra superparamagnetic iron oxide particles confirmed the specific affinity between P1C with $\alpha \nu \beta 3$ in Bel7402 human primary liver cancer cells in vitro (Lofblom et al., 2010) and in vivo (Wu et al., 2011). However, whether peptide binding to another integrin, such as $\alpha \operatorname{IIb} \beta 3$, will result in an antagonistic effect on platelet aggregation remains unclear.

In this study, a pentapeptide $\mathrm{P} 1 \mathrm{Cm}$ was first screened out from a series of truncated P1Cs with superior activity. The bioactivity of $\mathrm{P} 1 \mathrm{Cm}$ was evaluated in vitro and in vivo.

\section{MATERIALS AND METHODS}

\section{Peptide Synthesis}

The P1C peptide and its truncated forms were purchased from Scipeptide (Shanghai, China). The peptides were synthesized by the solid-phase method using a model 432A synthesizer (Applied Biosystems Inc., Foster City, CA; Angiolillo et al., 2004). Molecular mass was determined by electrospray mass spectrometry using LCMS-2010 (Shimadzu, Japan). The purity of the peptide was analyzed using a C18 reverse-phase HPLC (Wu et al., 2008). Table 1 presents a summary of all sequences of the truncated forms.

\section{Animals}

The animals were obtained from the Laboratory Animal Center, Science Academy of China (Shanghai, China) and raised in an SPF laboratory of a light/dark cycle of $12 \mathrm{~h} / 12 \mathrm{~h}$, with free access to food and water. Animal experiments were carried out according to a protocol approved by the Animal Care and Use Committee of Southeast University, China. To collect fresh animal samples, the animals were sacrificed through an intravenous injection of air after being anesthetized.
TABLE 1 | Anti-platelet activity of various peptides and Tirofiban.

\begin{tabular}{lc}
\hline Peptide/Tirofiban & IC50 $(\boldsymbol{\mu} \mathbf{M})$ \\
\hline IRTPKISKPIKFELSG (P1C) & 80 \\
IRTPK------(P1Cm) & 75 \\
$---I S K P I----$ & 442 \\
$------K F E L S G$ & 408 \\
IRTP------- & $>500$ \\
-RTPK------ & $>500$ \\
- RTP ------- & 377 \\
Tirofiban----- & 25
\end{tabular}

\section{Platelet Sample Preparation}

Blood samples from healthy adult donors who have never received antiplatelet treatment were collected and supplemented with $0.1 \mathrm{X}$ volumes of $3.8 \%$ trisodium citrate. After centrifugation at $1100 \mathrm{~g}$ for $15 \mathrm{~min}$ at $22^{\circ} \mathrm{C}$, the supernatant was collected as the platelet-poor plasma sample (Oyama et al., 2009). The pellets were transferred into a new tube and further purified by repeated re-suspension and centrifugation in Tyrode's albumin buffer three times. The cell pellets were finally re-suspended in the buffer as the platelet-rich plasma sample. Platelet count was performed using a whole-blood cell counter (Cysmex, USA). All samples were used within $6 \mathrm{~h}$ post-collection (Niu et al., 2012).

\section{Antiplatelet Aggregation Studies In vitro}

Platelet aggregation was measured by the turbidimetric assay using a platelet aggregation analyzer (LBY-NJ4, Beijing Precil Instrument Co. Ltd., China; Knight and Romano, 2005). A $300 \mu \mathrm{L}$ aliquot of platelet-rich plasma with $0-1.20 \mathrm{mM}$ peptide added was incubated under gentle stirring at $37^{\circ} \mathrm{C}$ for $5 \mathrm{~min}$ (Brossi et al., 2015). Adenosine diphosphate (ADP, $20 \mu \mathrm{mol} / \mathrm{L}$ ) or bovine thrombin (TH, 200 units/L) was added to induce platelet aggregation. The changes in transparence caused by platelet aggregation were recorded. The transparence of platelet samples with saline added was defined as $100 \%$. The transparence of the platelet sample with $\mathrm{ADP} / \mathrm{TH}$ added was defined as $0 \%$. The IC50 values of peptides for the blood sample were calculated as mean \pm SE.

The activity of P1Cm was further evaluated using the whole blood sample. Briefly, a $500 \mu \mathrm{L}$ aliquot of whole blood was placed in polystyrene tubes containing $0.009375-0.15 \mathrm{P} 1 \mathrm{Cm}$. The mixture was stirred at $37^{\circ} \mathrm{C}$ for $5 \mathrm{~min}$, followed by an addition of $60 \mu \mathrm{L}$ ADP or an equal volume of saline. Platelet counts were performed using a whole-blood cell counter (Cysmex, USA) after incubation at $37^{\circ} \mathrm{C}$ for $5 \mathrm{~min}$.

Blood smears were also prepared and stained using the Wright-Giemsa method (Nadal-Wollbold et al., 2010). Smear images were obtained with a Zeiss AxioVert 200M inverted light microscope (Carl Zeiss, Thornwood, NY, USA).

\section{P1Cm on the Coagulation Parameters and Its Acute In vivo Toxicity}

Plasma was collected after centrifugation at $1000 \times \mathrm{g}$ for $10 \mathrm{~min}$ at room temperature. An aliquot of $100 \mu \mathrm{l}$ plasma was 
supplemented with series concentrations of $\mathrm{P} 1 \mathrm{Cm}$, ranging from

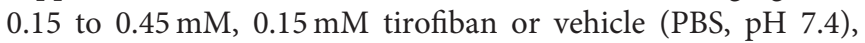
followed by an incubation of $5 \mathrm{~min}$ at $37^{\circ} \mathrm{C}$ before being sent to an ACL TOP Automatic Coagulation Analyzer (Beckman Coulter, USA; Wang et al., 2013). The coagulation parameters, such as activated partial thromboplastin time (APTT), prothrombin time (PT), and thrombin time (TT; Chen et al., 2013), were recorded, and each assay was performed in triplicate.

Kunming mice ( $n=6$ for each group) were intravenously injected with different concentrations of $\mathrm{P} 1 \mathrm{Cm}$, ranging from 0.075 to $6 \mathrm{mmol} / \mathrm{kg}$ (10x IC90), or tirofiban ranging from 0.025 to $2 \mathrm{mmol} / \mathrm{kg}$ (10x IC90). Blood pressure and breath were monitored after injection, and the mesentery were observed under microscope after the animals were sacrificed. Mortality within the next 3 days was recorded.

\section{Flow Cytometry}

Washed platelets $\left(1 \times 10^{6}\right.$ platelets $\left./ \mathrm{mL}\right)$ were pre-incubated with anti-human $\alpha$ IIb or $\beta 3$ monoclonal antibody $(5 \mu \mathrm{g} / \mathrm{mL}, \mathrm{R} \& \mathrm{D}$ Systems, Minneapolis, MN) for $10 \mathrm{~min}$ at room temperature in the presence of $100 \mathrm{U} / \mathrm{L}$ thrombin. After rinsing twice with PBS (Kander et al., 2014), the platelets were co-incubated with $5 \mu \mathrm{g} / \mathrm{mL}$ goat anti-mouse antibody-PE (Caltag Laboratories, USA) and/or $10 \mu \mathrm{g} / \mathrm{mL}$ FITC-conjugated peptide (FITC-P1Cm, synthesized by Science Peptide Bio-Technology Co, LTD., Shanghai, China) at $22^{\circ} \mathrm{C}$ for $30 \mathrm{~min}$ in darkness (Park et al., 2014). The incubations were then washed three times and fixed with $1 \%$ paraformaldehyde at $4^{\circ} \mathrm{C}$ (Kashiwagi et al., 2013) before flow cytometry analysis (Becton Dickinson, San Jose, CA, USA).

\section{Confocal Microscopy}

The platelet samples were prepared as previously reported (Mahdi et al., 2002; Elnager et al., 2014; Brzoska et al., 2015). In brief, human platelets $\left(1 \times 10^{6}\right.$ platelets $\left./ \mathrm{mL}\right)$ containing $0.1 \mathrm{mg} / \mathrm{mL}$ polylysine (Sigma) were transferred into a $35 \mathrm{~mm}$ cell culture dish (Cat no. 627860, Greiner Bio-One, Germany) followed by incubation for $30 \mathrm{~min}$ at room temperature. Thereafter, floating platelets were washed away with PBS $(\mathrm{pH}$ 7.4). The remaining platelets were treated with $100 \mathrm{U} / \mathrm{L}$ thrombin plus $5 \mu \mathrm{g} / \mathrm{mL}$ monoclonal antibody of anti-human $\alpha \mathrm{IIb}$ or $\beta 3$ for $30 \mathrm{~min}$ at room temperature. This step was followed by an addition of goat anti-mouse antibody-PE $(5 \mu \mathrm{g} / \mathrm{mL})$ and/or FITC-P1Cm $(10 \mu \mathrm{g} / \mathrm{mL})$ and incubation for $30 \mathrm{~min}$ in darkness at $4^{\circ} \mathrm{C}$. Finally, the platelets were washed twice with PBS and examined with a confocal laser scanning microscope (OLYMPUS-FV1000, Japan).

\section{P1Cm against Carotid Arterial Thrombosis}

Thirty male Wistar rats (250-300 g) were randomly divided into five groups: high-, middle-, and low-dose treated groups of P1Cm, a saline group, and a tirofiban group ( $n=6$ per group). The rats were operated on the right common carotid artery (Bird et al., 2008). Briefly, the rat was first anesthetized by an intraperitoneal injection of ketamine $(100 \mathrm{mg} / \mathrm{kg})$. The fascia was bluntly dissected to expose the right common carotid artery immediately after a dosage of $0.15,0.30$, or $0.45 \mathrm{mmoL} / \mathrm{kg}$ of $\mathrm{P} 1 \mathrm{Cm}, 0.15 \mathrm{mmoL} / \mathrm{kg}$ of tirofiban, or an equal volume of saline applied accordingly via tail vein injection. A $3 \times 4 \mathrm{~mm}$ piece of filter paper saturated with ferric chloride ( $10 \%$ solution) was placed under the right carotid artery for $20 \mathrm{~min}$ and then removed. Thrombus size and blood flow were detected by color Doppler flow imaging (CDFI) (13 MHz phased-array transducer, LOGIQ S6 Color Doppler Ultrasonographer, GE, USA; Chua et al., 2012). The animals were kept anesthetized during the CDFI measurements.

\section{Pathological Examination}

To obtain fresh samples immediately after measurement, the animals were sacrificed through an intravenous injection of air, and the injured carotid artery was collected for pathological examination. Carotid artery samples were fixed with $10 \%$ neutral buffered formalin and embedded in paraffin wax for routine histological analysis with hematoxylin and eosin staining. Morphometric analysis was carried out using a Leica Q500/W microscope (Leica Microsystems, Wetzlar, Germany). The antithrombosis effect was evaluated by calculating the percentage of carotid artery area covered by the thrombus (Hosokawa et al., 2011).

\section{Preventive Efficiency of $\mathrm{P} 1 \mathrm{Cm}$ in the Artery-Vein Shunt Model}

Fifty male Wistar rats (250-300 g) were randomly divided into five groups: high-, middle-, and low-dose groups of $\mathrm{P} 1 \mathrm{Cm}$, a saline group, and a tirofiban group ( $n=10$ per group). The animals were treated with the same concentrations as in the previous thrombosis model, $0.15,0.30$, or $0.45 \mathrm{mmoL} / \mathrm{kg}$ of P1Cm, $0.15 \mathrm{mmoL} / \mathrm{kg}$ of tirofiban, or an equal volume of saline by tail vein injection after being anesthetized by an intraperitoneal injection of ketamine (100 mg/kg). The operation was carried out as previously reported (Sato et al., 1998; Jing et al., 2011). Briefly, the right carotid and left jugular veins were separated. An $8 \mathrm{~cm}$ silk thread was placed inside a polyethylene tube filled with heparin sodium solution $(50 \mathrm{IU} / \mathrm{mL})$. The right carotid and left jugular veins were connected with the tube. One end of the tube containing the thread was inserted into the left jugular vein, and the other end was inserted into the right carotid artery. Blood was allowed to flow from the right carotid artery to the left jugular vein through the polyethylene tube for $15 \mathrm{~min}$. The tube was then removed, and the weight of the attached wet thrombus on the thread was measured immediately. The rats were sacrificed through an intravenous injection of air.

\section{Statistical Analyses}

Data are expressed as the mean \pm SD/SE and analyzed with SPSS 13.0 software. Significant differences between the control and experimental groups were analyzed by one-way analysis (ANOVA) followed by the least-significant difference (LSD) posthoc test. $P<0.05$ was considered statistically significant.

\section{Ethics Statement}

These studies were approved by IEC for Clinical Research of Zhongda Hospital, affiliated to Southeast University (Application number; 2013ZDSYLL109.0). 


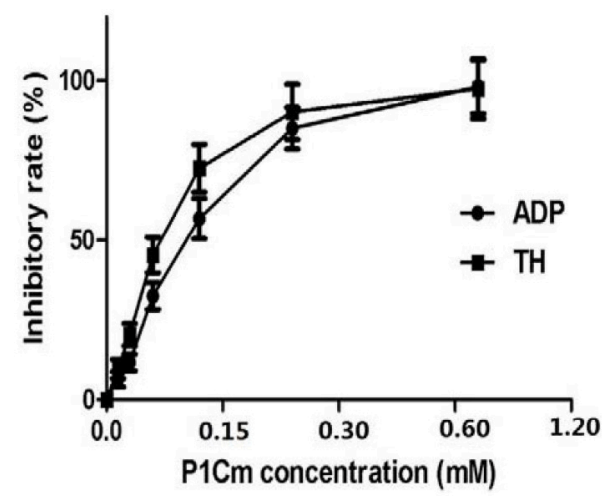

FIGURE 1 | Effects of P1Cm on ADP- and TH-induced platelet aggregation in human washed platelets. Platelets were pre-incubated with P1Cm (0-1.2 mM) for $5 \mathrm{~min}$, followed by an addition of ADP $(5 \mu \mathrm{M})$ or $\mathrm{TH}$ (200 units/L). Data are presented as mean $\pm \operatorname{SE}(n=6)$.

\section{RESULTS}

\section{P1Cm Activity against Platelet Aggregation}

The antiplatelet activities of $\mathrm{P} 1 \mathrm{Cm}$ or tirofiban were measured using the turbidimetric assay described above. The IC50 values of P1C and its truncated forms are summarized in Table 1. The smallest unit with antiplatelet activity was a 5-amino acid fragment (IRTPK) termed $\mathrm{P} 1 \mathrm{Cm}$. P1Cm exhibited superior activity to its parent and other truncated $\mathrm{P} 1 \mathrm{C}$ forms. P1Cm prevented platelet aggregation in a dose-dependent manner in the platelet-rich plasma samples (Figure 1). ADP induced platelet aggregation in the whole blood sample, as shown in Figure 2B. The whole blood sample pre-treated with $1 \mathrm{mg} / \mathrm{ml}$ $\mathrm{P} 1 \mathrm{Cm}$ showed a similar diffused distribution of platelets as negative control after the addition of ADP (Figures 2A,C). Respective $\mathrm{IC}_{50}$ values for $\mathrm{P} 1 \mathrm{Cm}$ inhibiting $\mathrm{ADP}$ - and $\mathrm{TH}$ induced platelet aggregation in the washed platelet samples were $145.9 \pm 12.13$ and $139.8 \pm 10.54 \mu \mathrm{M}$, and similar results were obtained with the whole blood sample (Figure 2, lower panel).

\section{Effects on Coagulation}

The FIB values were $3.61 \pm 0.44,3.82 \pm 0.37,3.94 \pm 0.45$, $4.07 \pm 0.39$, and $4.23 \pm 0.71 \mathrm{~g} / \mathrm{L}$ for the saline, P1Cm (low, middle, and high dosage), and tirofiban groups, respectively. The other three parameters are summarized in Figure 3. We observed that TT was postponed, whereas PT and APTT were as normal as the control after P1Cm treatment. P1Cm extended the TT at a dosage-dependent manner. No significant differences between groups of different dosages (high-, middle-, and low-dose groups; $P>0.05$ ) were found in PT, APTT, and FIB. In contrast, tirofiban apparently inhibited the coagulation and affected the parameters $(P<0.05)$.

\section{FITC-P1Cm Specifically Binds Integrin B3-Subunits of Platelets}

FITC and PE emitted green and red fluorescence, respectively. The platelets incubated with FITC-P1Cm alone showed green
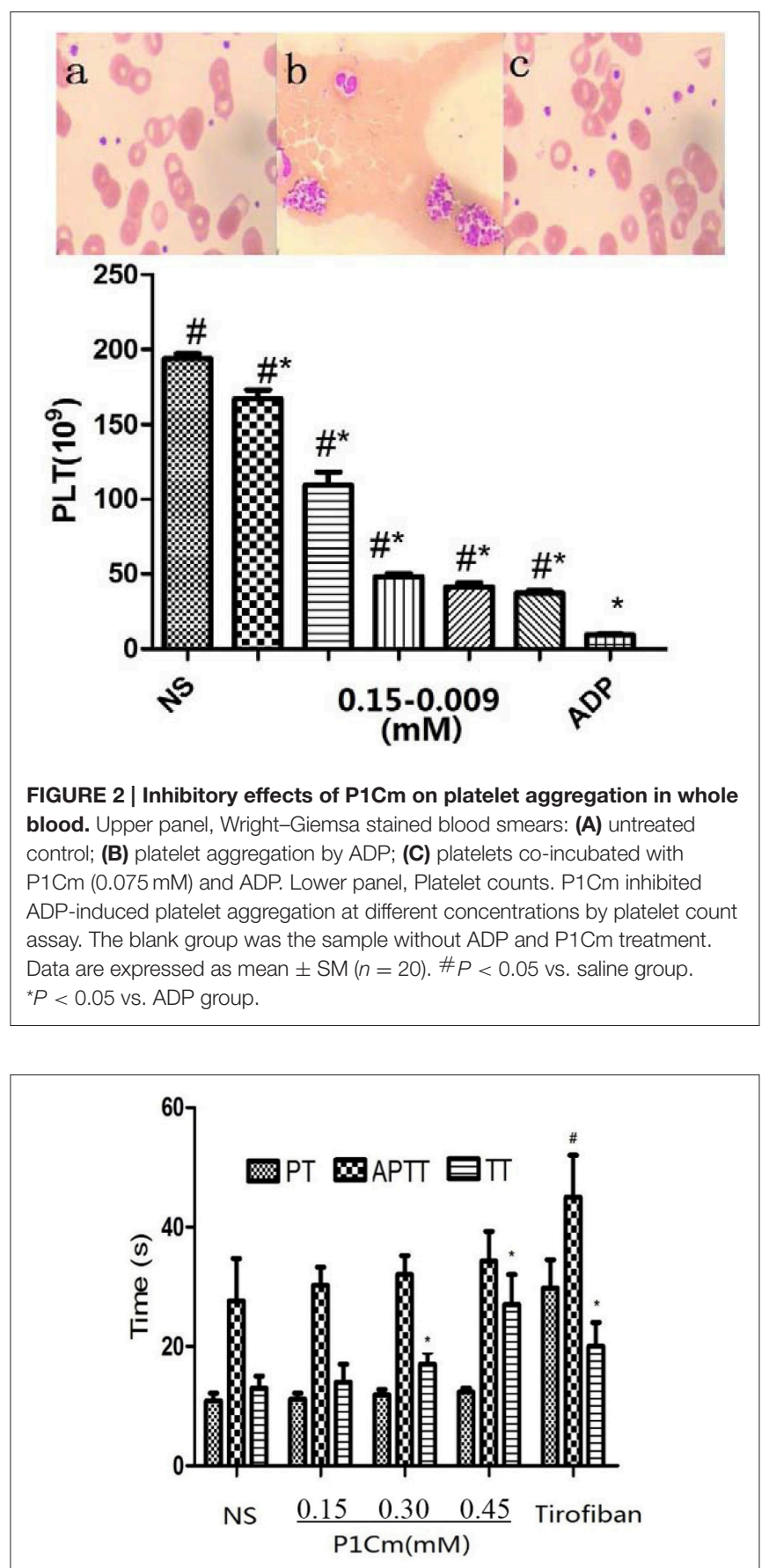

FIGURE 3 | Effect of P1Cm on coagulation. ${ }^{*} P<0.05$ significantly different from saline group. \#Significantly different from the rest of the groups. Data are expressed as mean $\pm \operatorname{SM}(n=6)$.

fluorescence (Figure 4E). The platelets gave off red fluorescence (Figure 4C) after subsequent treatment with anti- $\alpha$ IIb antibody or anti- $\beta 3$ antibody and goat anti-mouse antibody-PE. Only red fluorescence was present when the platelets were pre-incubated with $\beta 3$ antibody before FITC-P1Cm treatment (Figure 4D). In contrast, both green and red fluorescence were observed when 


$$
\text { 跑 }
$$


the platelets were subsequently treated with anti- $\alpha \mathrm{IIb}$ antibody, goat anti-mouse antibody-PE, and FITC-P1Cm (Figure 4B). No fluorescence signal was found in blank platelets without any treatment (Figure 4A).

Flow cytometric assay was performed to further confirm the affinity between $\mathrm{P} 1 \mathrm{Cm}$ with the $\beta 3$-subunit. Flow cytometry was used to determine the expression of $\alpha \mathrm{IIb} / \beta 3$-subunits of integrin and their interaction with FITC-labeled P1Cm. As shown in Figure 5, the $\alpha \mathrm{IIb}$ - and $\beta 3$-expression in platelets were $70.23 \pm 2.34$ and $50.18 \pm 4.86 \%$, respectively, with fluorescence signals located in the upper left quadrant (PE red; Figures 5A,B). The signal of the P1Cm-FITC bound platelets was $47.50 \pm$ $5.21 \%$, as shown in the lower right quadrant (FITC green; Figure 5C). No competition was observed between anti- $\alpha \mathrm{IIb}$ $\mathrm{mAb}$ with $\mathrm{P} 1 \mathrm{Cm}$ for platelet binding because the FITC green channel signal barely changed after receiving extra anti- $\alpha \mathrm{IIb} \mathrm{mAb}$ $(50.45 \pm 7.31$ vs. $47.50 \pm 5.21 \%)$. Some of the platelets were also double-stained (Figure 5D) after being co-incubated with P1Cm-FITC and anti- $\alpha$ IIb mAb. Consistent with the confocal microscopy result, the platelets pre-incubated with $\beta 3$ antibody no longer showed affinity with $\mathrm{P} 1 \mathrm{Cm}$, as shown in Figure 5E, indicating that anti- $\beta 3$ competed with $\mathrm{P} 1 \mathrm{Cm}$ for the same target. An untreated platelet sample was used as the blank control (Figure 5F).

\section{In vivo Antithrombotic Activity}

CDFI was used to detect the thrombosis size and evaluate the stenosis of the blood vessel through peak systolic flow velocity (PSFV). Anti-coagulation treatments significantly relieved the thrombosis. PSFV was negatively related to the vascular stenosis rate (VSR). When the vessel was blocked by thrombus, the flow rate increased as compensation for the supply of sufficient blood to the tissue. As expected, the highest $\mathrm{P} 1 \mathrm{Cm}$ dosage resulted in the slowest PSFV and the smallest VSR (Figure 6, Table 2), and its effects were very close to those of tirofiban on PSFV and VSR. The thrombosis areas in the carotid artery were $0.173 \pm 0.004$, $0.077 \pm 0.004$, and $0.0162 \pm 0.002 \mathrm{~cm}^{2}$ for the saline, low-dose, and middle-dose groups, respectively, but these were not detected in the high-dose group (Table 2, Figure S1 and Figure 6). P1Cm prevented thrombosis in a concentration-dependent manner, and a difference was observed within the $\mathrm{P} 1 \mathrm{Cm}$ groups in thrombosis size, VSR, and PSFV $(P<0.01)$. The vessel of the saline-treated model group was almost blocked without PSFV detection.

In the artery-vein shunt model, thrombus formation was prevented by antiplatelet treatment (Table 2). The thrombus weights evidently decreased by $9.33,20.04,43.35$, and $39.92 \%$ from the P1Cm (low $0.15 \mathrm{mM}$, middle $0.30 \mathrm{mM}$, and high dosage $0.45 \mathrm{mM}$ ) and tirofiban $0.15 \mathrm{mM}$ groups, respectively, in comparison with those from the saline group. The difference was statistically significant between the saline and treated groups $(P<0.05)$, except for the $\mathrm{P} 1 \mathrm{Cm}$ low-dose group $(P>0.05)$.

As shown in Table 3, both $\mathrm{P} 1 \mathrm{Cm}$ and tirofiban seemed safe when IC90 or a lower dosage was applied. P1Cm seemed to be safer than tirofiban when a higher dosage of 10X IC90 was applied. In contrast to the effects of $\mathrm{P} 1 \mathrm{Cm}$, the mice showed motor retardation after receiving 10X IC90 of tirofiban, and two mice died within $16 \mathrm{~h}$. Pathological examination showed the mesentery was characterized by local hemorrhage.

\section{DISCUSSION}

Aberrant platelet activation may cause thrombosis and eventually lead to serious vascular symptoms, such as cerebral stroke and myocardial infarction (De Luca, 2012). As an essential molecule during platelet activation and aggregation, $\alpha \operatorname{IIb} \beta 3$ is an excellent target for therapeutic intervention in thrombotic diseases (Nicholls et al., 2012; Diamond, 2013).

We reported on $\mathrm{P} 1 \mathrm{C}$ as an integrin-targeting peptide. In this study, a pentapeptide (IRTPK), P1Cm with antiplatelet aggregation, was screened out from a series of P1C truncated forms (Table 1). Numerous substances can target integrin, but few studies report on the discrimination of the two subunits of integrin for targeting. Through flow cytometry and confocal microscopy analyses, we demonstrated that $\mathrm{P} 1 \mathrm{Cm}$ was targeted on the $\beta 3$-subunit. Reports have indicated that the soluble form of the CD40 ligand, a tumor necrosis factor family member, mainly expressed on activated T-cells and platelets can mediate platelet stimulation by binding $\alpha \operatorname{IIb} \beta 3$ through a KGD domain (Prasad et al., 2003; Lonsdorf et al., 2012). The $\beta 3$-subunit is enriched on the platelet surface, an outcome that is the theoretical basis of the present study.

In the model of carotid thrombosis induced by ferric chloride, $\mathrm{P} 1 \mathrm{Cm}$ inhibited arterial thrombus formation in a concentration-dependent manner. The CDFI results illustrated that the thrombosis size and PSFV from the mid- and highdose groups were statistically different compared with those from the saline group $(P<0.05)$. Significant differences were also observed between the three $\mathrm{P} 1 \mathrm{Cm}$ dosage groups $(P<0.05)$. The result of pathological examination was in accordance with that of CDFI. The artery-vein shunt thrombosis model also showed the efficiency of P1Cm to reduce thrombus weight.

Our primary results indicated the weak effect of $\mathrm{P} 1 \mathrm{Cm}$ in vivo when used at the concentration of IC50; thus, 2x IC50 was chosen as the lowest concentration for the in vivo experiments. In this study, $\mathrm{P} 1 \mathrm{Cm}$ showed $43.35 \%$ thrombotic inhibition at a dose of $0.45 \mathrm{mmol} / \mathrm{kg}$, which was equal or even superior to the effect of tirofiban at the dose of $0.15 \mathrm{mmol} / \mathrm{kg}$, as shown by the in vivo experiment. Importantly, $\mathrm{P} 1 \mathrm{Cm}$ seemed to be safer than tirofiban, as shown by the coagulation assay and in vivo toxicity evaluation. $\mathrm{P} 1 \mathrm{Cm}$ had less effect on the extrinsic and intrinsic coagulation cascades. PT and APTT are usually used to evaluate the extrinsic and intrinsic coagulation pathways, respectively. In contrast to tirofiban, no significant difference in PT or APTT was found between the P1Cm-treated groups (high, middle, and low doses), and the saline group $(P>0.05)$ in this study. The lethal dose of P1Cm overreached 10X IC 90 of P1Cm, whereas tirofiban caused mouse death at the dosage of 10X IC90. Histological examination indicated that tirofiban caused capillary vessel hemorrhage.

In the last decades, millions of thrombosis patients have benefited from antiplatelet treatments, including $\alpha \operatorname{IIb} \beta 3$ inhibitors, and most have survived. Nevertheless, these patients 

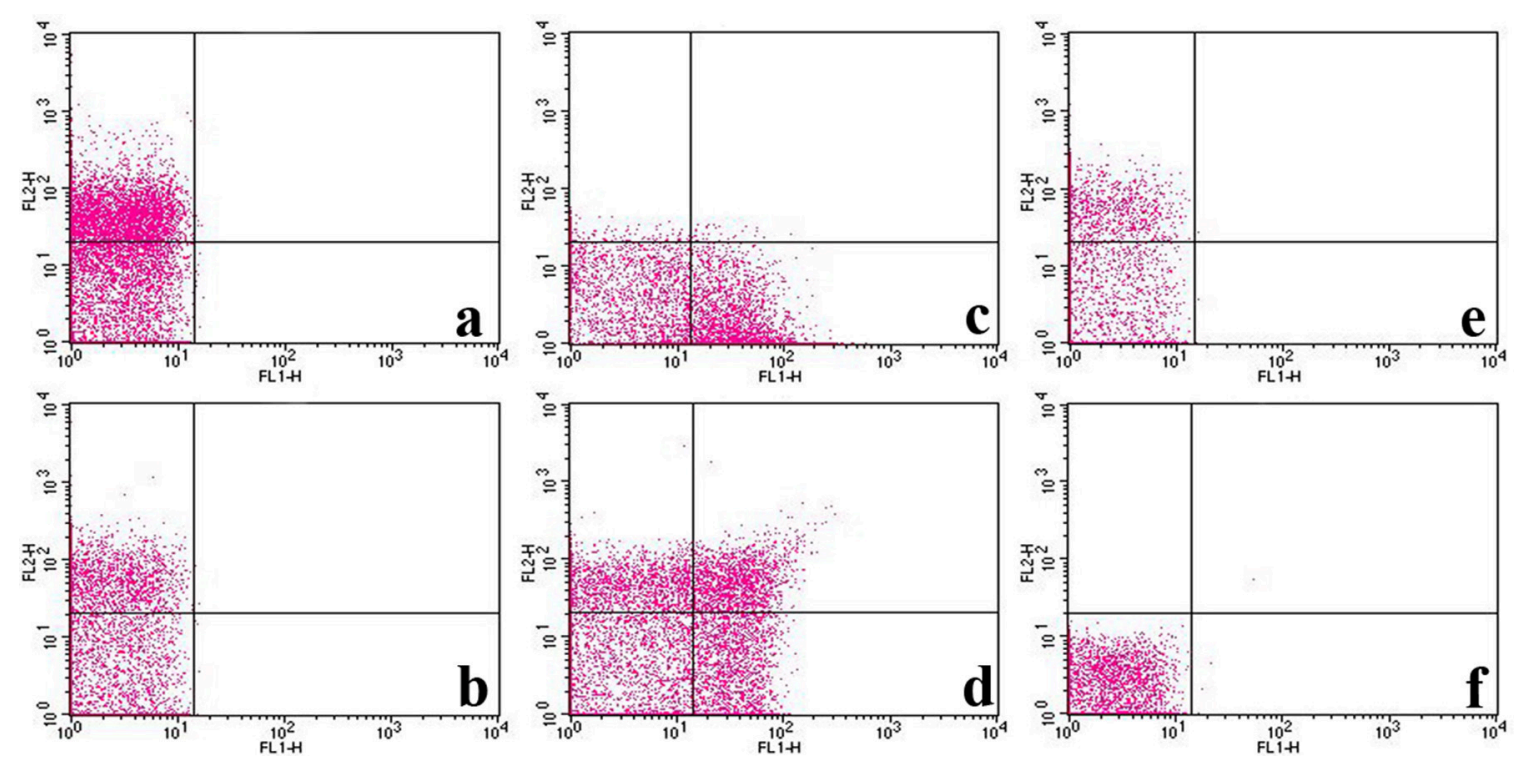

FIGURE 5 | FITC-labeled P1Cm specifically binds to platelets using flow cytometry. (A) Platelet sample treated with anti-allb antibody and goat anti-mouse antibody-PE successively. (B) Sample treated with anti- $\beta 3$ antibody and goat anti-mouse antibody-PE. (C) Sample treated with FITC-P1Cm. (D) Sample pre-treated

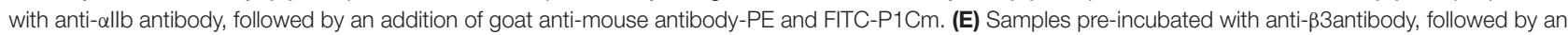
addition of goat anti-mouse antibody-PE and FITC-P1Cm. (F) Untreated platelets.

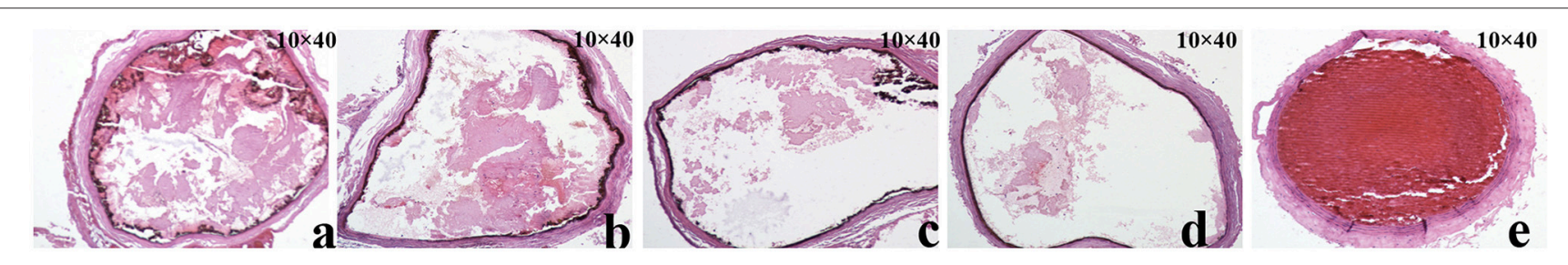

FIGURE 6 | Pathological images of thrombosis in carotid artery. Slices of carotid artery after rats received (A) $0.15 \mathrm{mmol} / \mathrm{kg} \mathrm{P1Cm}$, (B) $0.30 \mathrm{mmol} / \mathrm{kg} \mathrm{P1Cm}$, or (C) $0.45 \mathrm{mmol} / \mathrm{kgP} 1 \mathrm{Cm}$, (D) $0.15 \mathrm{mmol} / \mathrm{kg}$ tirofiban, or (E) saline.

TABLE 2 | Effects of P1Cm on the carotid thrombosis model and the artery-vein shunt model.

\begin{tabular}{|c|c|c|c|c|}
\hline Groups (mmol/kg) & PSFV (cm/s) & SIZE/area $\left(\mathrm{cm}^{2}\right)$ & EMW (mg) & VSR (\%) \\
\hline Saline & None $^{\#}$ & $0.173 \pm 0.004$ & $46.07 \pm 4.46$ & $83.95 \pm 5.91$ \\
\hline Tirofiban 0.15 & $84.2 \pm 3.50^{*}$ & $0.007 \pm 0.001^{\star}$ & $27.51 \pm 4.03^{\star}$ & $28.25 \pm 5.83^{\star}$ \\
\hline $\mathrm{P} 1 \mathrm{Cm} 0.15$ & $109.6 \pm 10.52^{*}$ & $0.077 \pm 0.004^{\star}$ & $41.77 \pm 5.87$ & $66.05 \pm 6.99$ \\
\hline $\mathrm{P} 1 \mathrm{Cm} 0.30$ & $97.3 \pm 3.22^{\star}$ & $0.016 \pm 0.002^{*}$ & $36.83 \pm 4.86^{\star}$ & $50.00 \pm 8.50^{\star}$ \\
\hline $\mathrm{P} 1 \mathrm{Cm} 0.45$ & $81.5 \pm 5.59^{\star}$ & $\mathrm{None}^{+}$ & $26.10 \pm 4.74^{\star}$ & $31.84 \pm 12.72^{*}$ \\
\hline
\end{tabular}

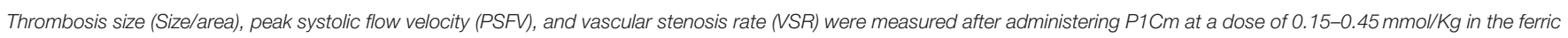

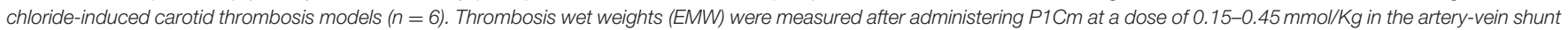
models $(n=10)$. Data are expressed as mean $\pm S D$. \#Blood vessel was almost blocked by thrombus. ${ }^{+}$No thrombus was detected. ${ }^{\star} P<0.05$ vs. saline groups.

were confronted with another risk of intrinsic bleeding, which raised the paradox of the antiplatelet substance being able to inhibit the thrombosis efficiently but not affecting the normal coagulation. Given that platelets play an essential role in hemostasis and thrombosis (Abtahian et al., 2015), balancing platelet function is effective in preventing thrombosis and seems to be the key to reducing bleeding risk during antiplatelet therapy (Viswanathan et al., 2012). In fact, because of the high risk of bleeding due to their unconscionable high affinity to $\alpha \operatorname{IIb} \beta 3$, receptor inhibitors are no longer considered clinically safe (Elcioglu et al., 2012). The occurrence of thrombocytopenia and the increased risk of major and minor bleeding complications 
TABLE 3 | Acute toxicity of P1Cm and Tirofiban in mice $(n=6)$.

\begin{tabular}{lccc}
\hline Dosage/mortality & IC50 & IC90 & 10x IC90 \\
\hline P1Cm & 0 & 0 & 0 \\
Tirofiban & 0 & $0 / 6$ & $2 / 6$ \\
\hline
\end{tabular}

have become main concerns. Thrombocytopenia is frequently reported for abciximab, eptifibatide, and tirofiban treatment, and bleeding complications are regarded as major determinants of clinical outcomes in percutaneous coronary interventions (Huynh et al., 2005). However, $\alpha \operatorname{IIb} \beta 3$ inhibitors are still widely used to prevent periprocedural thrombosis during PCI. Thus, the need for novel, safe antithrombotic drugs is urgent.

Balancing the risk of thrombocytopenia or bleeding complications with the curative effect of anti-coagulants is a difficult undertaking (Ji and Hou, 2011). The key lies in the affinity of $\mathrm{P} 1 \mathrm{Cm}$ to $\alpha \mathrm{IIb} \beta 3$. One possible solution is to administer P1Cm by continuous infusion. P1Cm may alleviate the risk of thrombocytopenia and bleeding complications, as it shows relatively smaller efficiency than other $\alpha \operatorname{IIb} \beta 3$ inhibitors. Cyclization is another option to improve the affinity. We plan to focus on modifying the structure to further develop P1Cm.

In addition, unlike eptifibatide from snake venom, $\mathrm{P} 1 \mathrm{Cm}$ is a small peptide derived from human connective tissue growth factor, and it is expected to cause very limited allergic or hypersensitivity reactions during or after infusion. Compared with antibodies such as abciximab, $\mathrm{P} 1 \mathrm{Cm}$ is a small peptide of 5 -amino acid that results in less stereo-specific blockades, and possibly a high drug-to-receptor ratio. In reference to the results on toxicity in vivo (Table 3), the safety of P1Cm is apparently superior to, but not as effective as, that of tirofiban.

In conclusion, $\mathrm{P} 1 \mathrm{Cm}$ can clearly inhibit ADP- or TH-induced human platelet aggregation in vitro in a concentration-dependent

\section{REFERENCES}

Abtahian, F., Waldo, S., and Jang, I. K. (2015). Comparison of heparin and bivalirudin in patients undergoing percutaneous coronary intervention without use of glycoprotein IIb/IIIa inhibitors. Catheter. Cardiovasc. Interv. 86, 390-396. doi: 10.1002/ccd.25911

Angiolillo, D. J., Fernandez-Ortiz, A., Bernardo, E., Ramirez, C., Sabate, M., Hernandez-Antolin, R., et al. (2004). Is a $300 \mathrm{mg}$ clopidogrel loading dose sufficient to inhibit platelet function early after coronary stenting? A platelet function profile study. J. Invasive Cardiol. 16, 325-329.

Armstrong, P. C., and Peter, K. (2012). GPIIb/IIIa inhibitors: from bench to bedside and back to bench again. Thromb. Haemost. 107, 808-814. doi: 10.1160/TH11-10-0727

Bird, J. E., Giancarli, M. R., Allegretto, N., Barbera, F., Wong, P., Schumacher, W. A., et al. (2008). Prediction of the therapeutic index of marketed anticoagulants and anti-platelet agents by guinea pig models of thrombosis and hemostasis. Thromb. Res. 123, 146-158. doi: 10.1016/j.thromres.2008.03.010

Brossi, P. M., Moreira, J. J., Machado, T. S., and Baccarin, R. Y. (2015). Platelet-rich plasma in orthopedic therapy: a comparative systematic review of clinical and experimental data in equine and human musculoskeletal lesions. BMC Vet. Res. 11:98. doi: 10.1186/s12917-015-0403-z manner by specifically binding to the $\beta_{3}$-subunit. Compared to tirofiban, $\mathrm{P} 1 \mathrm{Cm}$ seems to be safe when a much higher dosage is applied in vivo, with less effect on the extrinsic and intrinsic coagulation pathways. Further experiments using two rat thrombosis models demonstrated that $\mathrm{P} 1 \mathrm{Cm}$ is also an excellent antithrombotic agent in vivo. $\mathrm{P} 1 \mathrm{Cm}$ is a promising antithrombotic alternative to currently available treatments.

\section{AUTHOR CONTRIBUTIONS}

QQ and GW performed most of the experiments and prepared the manuscript. YL assisted with the rat experiments. XF revised the manuscript. GW and NL were the group leaders from two independent groups offering supervision and financial support.

\section{ACKNOWLEDGMENTS}

This work was supported by the National Natural Science Foundation of China (Nos. 30970809 and 81271636), the Natural Science Foundation of Jiangsu Province (No. BK2009274), the Special Fund of Clinical Medicine, Jiangsu Province, China (No. BL2012063), and the Fundamental Research Funds for the Central Universities (Nos. 2013YX006 and 2242014K40006).

\section{SUPPLEMENTARY MATERIAL}

The Supplementary Material for this article can be found online at: http://journal.frontiersin.org/article/10.3389/fphar. 2016.00049

Figure S1 | PSFV and thrombosis size in ferric chloride-induced carotid thrombosis models detected by CDFI. Rats were measured after receiving (A) $0.15 \mathrm{mmol} / \mathrm{Kg}$, (B) $0.30 \mathrm{mmol} / \mathrm{Kg}$ or (C) $0.45 \mathrm{mmol} / \mathrm{Kg} \mathrm{P} 1 \mathrm{Cm}$, or (D)

$0.15 \mathrm{mmol} / \mathrm{Kg}$ tirofiban. Saline (E) was used as negative control.
Brzoska, T., Tanaka-Murakami, A., Suzuki, Y., Sano, H., Kanayama, N., and Urano, T. (2015). Endogenously generated plasmin at the vascular wall injury site amplifies lysine binding site-dependent plasminogen accumulation in microthrombi. PLoS ONE 10:e0122196. doi: 10.1371/journal.pone.01 22196

Chen, T. Q., Chen, C. C., Wang, J. X., Chen, W. F., Gu, X. M., Xu, R. S., et al. (2013). Effect of glutathione on blood coagulation function. J. Exp. Hematol. 21, 1612-1616. doi: 10.7534/j.issn.1009-2137.2013.06.046

Chua, H. C., Lim, T., Teo, B. C., Phua, Z., and Eng, J. (2012). Freefloating thrombus of the carotid artery detected on carotid ultrasound in patients with cerebral infarcts: a 10-year study. Ann. Acad. Med. Singapore 41, 420-424. Available online at: http://www.annals.edu.sg/pdf/ 41VolNo9Sep2012/V41N9p420.pdf

Cornel, J. H., Tricoci, P., Lokhnygina, Y., Moliterno, D. J., Wallentin, L., Armstrong, P. W., et al. (2015). Glycoprotein IIb/IIIa receptor inhibitors in combination with Vorapaxar, a platelet thrombin receptor antagonist, among patients with Non-ST-Segment Elevation Acute Coronary Syndromes (from the TRACER Trial). Am. J. Cardiol. 115, 1325-1332. doi: 10.1016/j.amjcard.2015.02.043

De Luca, G. (2012). Glycoprotein IIb-IIIa inhibitors. Cardiovasc. Ther. 30, e242e254. doi: 10.1111/j.1755-5922.2011.00293.x 
Diamond, S. L. (2013). Systems biology of coagulation. J. Thromb. Haemost. 11(Suppl. 1), 224-232. doi: 10.1111/jth.12220

Di Mario, C. (2014). Rise and fall of glycoprotein IIb-IIIa inhibitors in ST-segment elevation myocardial infarction. Heart 100, 823-824. doi: 10.1136/heartjnl2013-305374

Elcioglu, O. C., Ozkok, A., Akpinar, T. S., Tufan, F., Sezer, M., Umman, S., et al. (2012). Severe thrombocytopenia and alveolar hemorrhage represent two types of bleeding tendency during tirofiban treatment: case report and literature review. Int. J. Hematol. 96, 370-375. doi: 10.1007/s12185-012-1133-7

Elnager, A., Abdullah, W. Z., Hassan, R., Idris, Z., Wan Arfah, N., Sulaiman, S. A., et al. (2014). In vitro whole blood clot lysis for fibrinolytic activity study using d-dimer and confocal microscopy. Adv. Hematol. 2014:814684. doi: $10.1155 / 2014 / 814684$

Gao, R., and Brigstock, D. R. (2004). Connective tissue growth factor (CCN2) induces adhesion of rat activated hepatic stellate cells by binding of its Cterminal domain to integrin alpha(v)beta(3) and heparan sulfate proteoglycan. J. Biol. Chem. 279, 8848-8855. doi: 10.1074/jbc.M313204200

Hanna, E. B., Rao, S. V., Manoukian, S. V., and Saucedo, J. F. (2010). The evolving role of glycoprotein IIb/IIIa inhibitors in the setting of percutaneous coronary intervention strategies to minimize bleeding risk and optimize outcomes. JACC. Cardiovasc. Interv. 3, 1209-1219. doi: 10.1016/j.jcin.2010.09.015

Hechler, B., Freund, M., Alame, G., Leguay, C., Gaertner, S., Cazenave, J. P., et al. (2011). The antithrombotic activity of EP224283, a neutralizable dual factor Xa inhibitor/glycoprotein IIbIIIa antagonist, exceeds that of the coadministered parent compounds. J. Pharmacol. Exp. Ther. 338, 412-420. doi: 10.1124/jpet.111.181321

Hosokawa, K., Ishibashi-Ueda, H., Kishi, T., Nakanishi, N., Kyotani, S., and Ogino, H. (2011). Histopathological multiple recanalized lesion is critical element of outcome after pulmonary thromboendarterectomy. Int. Heart J. 52, 377-381. doi: 10.1536/ihj.52.377

Huynh, T., Piazza, N., DiBattiste, P. M., Snapinn, S. M., Wan, Y., Pharand, C., et al. (2005). Analysis of bleeding complications associated with glycoprotein IIb/IIIa receptors blockade in patients with high-risk acute coronary syndromes: insights from the PRISM-PLUS study. Int. J. Cardiol. 100, 73-78. doi: 10.1016/j.ijcard.2004.07.014

Ji, X., and Hou, M. (2011). Novel agents for anti-platelet therapy. J. Hematol. Oncol. 4:44. doi: 10.1186/1756-8722-4-44

Jing, B. B., Li, Y. X., Zhang, H., Ren, S. T., Wang, M., Li, Y. P., et al. (2011). Antithrombotic activity of Z4A5, a new platelet glycoprotein IIb/IIIa receptor antagonist evaluated in a rabbit arteriovenous shunt thrombosis model. Thromb. Res. 128, 463-469. doi: 10.1016/j.thromres.2011.08.003

Kander, T., Tanaka, K. A., Norstrom, E., Persson, J., and Schott, U. (2014). The effect and duration of prophylactic platelet transfusions before insertion of a central venous catheter in patients with bone marrow failure evaluated with point-of-care methods and flow cytometry. Anesth. Analg. 119, 882-890. doi: 10.1213/ANE.0000000000000259

Kashiwagi, H., Kunishima, S., Kiyomizu, K., Amano, Y., Shimada, H., Morishita, M., et al. (2013). Demonstration of novel gain-of-function mutations of alphaIIbbeta3: association with macrothrombocytopenia and glanzmann thrombasthenia-like phenotype. Mol. Genet. Genomic Med. 1, 77-86. doi: $10.1002 / \mathrm{mgg} 3.9$

Knight, L. C., and Romano, J. E. (2005). Functional expression of bitistatin, a disintegrin with potential use in molecular imaging of thromboembolic disease. Protein Expr. Purif. 39, 307-319. doi: 10.1016/j.pep.2004.11.005

Lefkovits, J., Plow, E. F., and Topol, E. J. (1995). Platelet glycoprotein IIb/IIIa receptors in cardiovascular medicine. N. Engl. J. Med. 332, 1553-1559. doi: 10.1056/NEJM199506083322306

Liu, J., Jackson, C. W., Gruppo, R. A., Jennings, L. K., and Gartner, T. K. (2005). The beta3 subunit of the integrin alphaIIbbeta3 regulates alphalIb-mediated outside-in signaling. Blood 105, 4345-4352. doi: 10.1182/blood-2004-07-2718

Lofblom, J., Feldwisch, J., Tolmachev, V., Carlsson, J., Stahl, S., and Frejd, F. Y. (2010). Affibody molecules: engineered proteins for therapeutic, diagnostic and biotechnological applications. FEBS Lett. 584, 2670-2680. doi: 10.1016/j.febslet.2010.04.014

Lonsdorf, A. S., Kramer, B. F., Fahrleitner, M., Schonberger, T., Gnerlich, S., Ring, S., et al. (2012). Engagement of alphaIIbbeta3 (GPIIb/IIIa) with alphanubeta3 integrin mediates interaction of melanoma cells with platelets: a connection to hematogenous metastasis. J. Biol. Chem. 287, 2168-2178. doi: 10.1074/jbc.M111.269811

Mahdi, F., Madar, Z. S., Figueroa, C. D., and Schmaier, A. H. (2002). Factor XII interacts with the multiprotein assembly of urokinase plasminogen activator receptor, gC1qR, and cytokeratin 1 on endothelial cell membranes. Blood 99, 3585-3596. doi: 10.1182/blood.V99.10.3585

Nadal-Wollbold, F., Bocquet, A., Bourbon, T., Letienne, R., and Le Grand, B. (2010). Protease-activated receptor 1 antagonists prevent platelet aggregation and adhesion without affecting thrombin time. Eur. J. Pharmacol. 644, 188-194. doi: 10.1016/j.ejphar.2010.07.013

Nicholls, S. J., Cavender, M. A., Kastelein, J. J., Schwartz, G., Waters, D. D., Rosenson, R. S., et al. (2012). Inhibition of secretory phospholipase A(2) in patients with acute coronary syndromes: rationale and design of the vascular inflammation suppression to treat acute coronary syndrome for 16 weeks (VISTA-16) trial. Cardiovasc. Drugs Ther. 26, 71-75. doi: 10.1007/s10557-0116358-9

Niu, H., Chen, X., Gruppo, R. A., Li, D., Wang, Y., Zhang, L., et al. (2012). Integrin alphaIIb-mediated PI3K/Akt activation in platelets. PLOS ONE 7:e47356. doi: 10.1371/journal.pone.0047356

Oyama, E., Furudate, N., Senuki, K., and Takahashi, H. (2009). Purification and characterization of a new platelet aggregation inhibitor with dissociative effect on ADP-induced platelet aggregation, from the venom of Protobothrops elegans (Sakishima-habu). Toxicon 53, 706-712. doi: 10.1016/j.toxicon.2009.02.016

Park, S. H., Park, C. J., Kim, M. J., Han, M. Y., Lee, B. R., Cho, Y. U., et al. (2014). The Sysmex XN-2000 hematology autoanalyzer provides a highly accurate platelet count than the former Sysmex XE-2100 system based on comparison with the CD41/CD61 immunoplatelet reference method of flow cytometry. Ann. Lab. Med. 34, 471-474. doi: 10.3343/alm.2014.34.6.471

Prasad, K. S., Andre, P., He, M., Bao, M., Manganello, J., and Phillips, D. R. (2003). Soluble CD40 ligand induces beta3 integrin tyrosine phosphorylation and triggers platelet activation by outside-in signaling. Proc. Natl. Acad. Sci. U.S.A. 100, 12367-12371. doi: 10.1073/pnas.2032886100

Rodrigues, A. N., Abreu, G. R., Resende, R. S., Goncalves, W. L., and Gouvea, S. A. (2013). Cardiovascular risk factor investigation: a pediatric issue. Int. J. Gen. Med. 6, 57-66. doi: 10.2147/IJGM.S41480

Sato, K., Kawasaki, T., Hisamichi, N., Taniuchi, Y., Hirayama, F., Koshio, H. et al. (1998). Antithrombotic effects of YM-60828 in three thrombosis models in guinea pigs. Eur. J. Pharmacol. 350, 87-91. doi: 10.1016/S0014-2999(98) 00328-8

Viswanathan, G., Kidambi, A., Nelson, A., Mayurathan, G., Hardy, J., Kesteven, P., et al. (2012). Glycoprotein IIb/IIIa inhibitor associated severe thrombocytopenia in patients with coronary artery disease: clinical course and outcomes. Platelets 23, 224-228. doi: 10.3109/09537104.2011.604804

Wang, P., Wang, Q., Luo, C., Tan, C., and Yuan, X. (2013). Iridoid glycosides extracted from zhizi (fructus gardeniae) decrease collagen-induced platelet aggregation and reduce carotid artery thrombosis in an in vivo rat model. J. Tradit. Chin. Med. 33, 531-534. doi: 10.1016/S0254-6272(13)60160-0

Wu, G. Q., Li, L. X., Ding, J. X., Wen, L. Z., and Shen, Z. L. (2008). High-level expression and novel purification strategy of recombinant thanatin analog in Escherichia coli. Curr. Microbiol. 57, 95-101. doi: 10.1007/s00284-008-9106-Z

Wu, G., Wang, X., Deng, G., Wu, L., Ju, S., Teng, G., et al. (2011). Novel peptide targeting integrin alphavbeta3-rich tumor cells by magnetic resonance imaging. J. Magn. Reson. Imaging 34, 395-402. doi: 10.1002/jmri.22620

Conflict of Interest Statement: The authors declare that the research was conducted in the absence of any commercial or financial relationships that could be construed as a potential conflict of interest.

Copyright $\odot 2016$ Qu, Liu, Yan, Fan, Liu and Wu. This is an open-access article distributed under the terms of the Creative Commons Attribution License (CC BY). The use, distribution or reproduction in other forums is permitted, provided the original author(s) or licensor are credited and that the original publication in this journal is cited, in accordance with accepted academic practice. No use, distribution or reproduction is permitted which does not comply with these terms. 